Wahrscheinlichkeitsrechnung und Mathematische Statistik

LEXIKON DER STOCHASTIK 



\section{Wahrscheinlichkeitsrechnung und Mathematische Statistik}

Herausgegeben von

Prof. Dr. P. H. MUULLER

Technische Universität Dresden

\section{LEXIKON DER STOCHASTIK}

2., bearbeitete und wesentlich erweiterte Auflage

AKADEMIE-VERLAG • BERLIN

1975 
Autorenkollektiv unter Federführung

von Prof. Dr. rer. nat. habil. P. HeInz MüLler

Dr. phil. Helmot Ebersberger

Dr. rer. nat. HEINZ GILLERT

Dr. rer. nat. UwE KüCHLER

Dr. rer. nat. habil. RoLF KÜHNE

Prof. Dr. rer. nat. habil. Heinz Langer

Dr. rer. nat. Dr. paed. Gert MaIBAUM

Dr. rer. nat. Peter Neumani

Dr. sc. nat. VolkeR NolLAU

Dr. rer. nat. LothaR PARTzSCH

Dr. rer. nat. Franz SchMidT

Dr. rer. nat. Regina Storm

Prof. Dr. rer. nat. habil. Wolfgang WinkLeR

Dr. rer. nat. GISELA WITTWER

Erschienen im Akademie-Verlag, 108 Berlin, Leipziger Str. 3-4

(c) Akademie-Verlag, Berlin, 1975

Lizenznummer: $202 \cdot 100 / 411 / 75$

Gesamtherstellung:VEB Druckerei ,Thomas Müntzer“, 582 Bad Langensalza

Bestellnummer: 7621816 (5771) · LSV 1077

Printed in GDR

EVP 29,- 